The Promise of Inclusion for Female Student Health

Kate D. Romero \& Kymberlee M. O’Brien

Keywords: inclusion, diversity, women's health, stress

Acknowledgments. We are grateful to acknowledge the award of this research by the Society of Personality and Social Psychology to the second author. 


\begin{abstract}
Despite extensive inclusion and diversity initiatives, females do not feel valued or included and still report higher stress, discrimination and microaggressions than males. Cumulative effects of social devaluation on health were examined for students at a STEM University. A sample of 292 undergraduates were asked about daily and chronic experiences of inclusion using surveys assessing personal perceived stress and subtle and overt social devaluation. Females reported significantly higher microaggressions and perceived stress, associated with lower physical and mental health. Females in high social devaluation (SD) reported lower total well-being (TWB) across several domains. An exploratory factor analyses examined factor loadings on perceptions of devaluation and extracted three factors; results showed that females and males perceive the poor treatments for markedly different reasons. Stress, low sense of control, objectification, and lack of positive exemplars varied by sex. These data suggest persistent implicit biases remain entrenched for females in STEM. This was unexpected since multiple early inclusion interventions exist. Inclusion initiatives may need to be reviewed specifically to address implicit attitudes and internalized acquiescence, training female students to explicitly interface with such experiences.
\end{abstract}


Most universities are highly invested in increasing diversity and inclusion in STEM fields, although limited information is publicized on specific interventions and practices offered. Most of the interventions support diversity, yet tangible training and dialogue remain vague. The data presented here show that females experience implicit devaluation and future interventions may need to address student's experiences more explicitly.

Statements of commitment to inclusion are common. For example, many universities publish their diversity and inclusion items online, including appointing a chief diversity officer to ensure implementation of practices involving recruitment and retention of students, administrators, and faculty. Administration states it will ensure campus diversity and inclusion plans are in place to meet its commitments and ensure diverse candidate pools. These are laudable aims; however, the implementation of practices and student experiences appear uncoupled. While there are overt statements of support for female and minority students, particularly in STEM fields, whether plan elements effectively address experiences of exclusion/devaluation is not explicit.

Interventions to promote equality in STEM are set in place at the federal level, however their effectiveness is also unclear since attrition rates for women in STEM remain high (Xu, 2017). According to Title IX, sex bias prevents girls and women from pursuing a STEM education, although among high schoolers, interest and achievement in STEM are at an all-time high. Initiatives for females in STEM have been increasing in recent years, however there is still discrimination in the workplace. The succeeding initiatives need to be supplemented with outreach and retention programs, family-friendly faculty policies, and strong adherence to and monitoring of regulatory compliance in schools, colleges, and research institutions throughout the country (Women and STEM, 2017). Since Congress enacted Title IX in 1972 to prohibit sex 
discrimination under any activity or educational program receiving any kind of federal finding, there has been a tenfold increase in women employed in academia for STEM fields. In 2013, women comprised $24 \%$ of full professors, $38 \%$ of associate professors, and $45 \%$ of assistant professors (Women and STEM, 2017). In 2017, a new chapter was included titled "Title IX and STEM," as well as a chapter on Career and Technical Education (Title IX and STEM, n.d.). Provisions to Title IX include extensive initiatives to continue encouraging equality in STEM by supporting female students, encouraging universities to evaluate admissions to prevent discrimination, and by offering tenure-track eligibility and other options for women with young children. These are only a few of the many initiatives published as part of Title IX (Women and STEM, 2017), yet the translation of legislation to addressing implicit negative biases may not be as effective.

Despite women's increased representation in STEM after the passage of Title IX, information is still lacking about initiatives and interventions to prevent or address unconscious bias. A study conducted at the University of Mississippi found that microaggressions within the college campus commonly occurred in living spaces, Greek organizations, in the classroom, at the student union, and at the recreational center, but the university lacked strategies for preventing this discrimination (Johnson et al., 2018). With the dangerous health implications stemming from subtle social devaluation, it is critical for STEM institutions to formulate more effective ways of helping female students, staff, and faculty handle microaggressions.

\section{Microaggressions in STEM}

"Microaggression" is now a well-known term that is defined as a more subtle form of social devaluation, compared to overt sexism or racism; these negative social interactions have been identified as stressful and associated with several domains of immutable identity including 
sex, race/ethnicity, and sexual orientation (Balsam et al., 2011; Sue, 2007; Smith, Hung, \& Franklin, 2011). Microaggressions have been highlighted in the literature and are defined as subtle or ambiguous social devaluing statements that are common to the receiver (Sue, 2007; Nadal, 2011). They can be classified as daily indignities that are difficult to process given ambiguous subtext. Moreover, Wong et al., (2014) suggest that these innocuous or minor interactions may trigger a negative stress reaction, while the perpetrator may perceive these interactions as complimentary ("You are good at math for a female"). Adding to this, the overall negativity and impact on health for the receiver are dismissed as harmless or minimized, or the victim is derogated (e.g., they are being "too sensitive"). While overt discrimination experiences are established as chronic stressors and are considered a major contributor to poor physical and mental health and higher mortality rates for disadvantaged populations (Adler \& Rehkopf, 2008; Troxel, et al., 2003; O’Brien, Meyer, Tronick, \& Moore, 2017), not yet explicated is the cumulative psychological stress for recipients experiencing subtle sexist and perhaps chronic devaluing interactions on overall health and functioning. Growing evidence suggests that social devaluation can have cumulative negative effects for certain individuals (Sue, 2007; Nadal, 2011).

Numerous studies have investigated overt discrimination as physiological acute or chronic stressors (e.g., O’Brien, Meyer, Tronick, \& Moore, 2017) that may also be tapping into global experiences of social devaluation, particularly for social identity features which are immutable. Moreover, overt discrimination and perceived expectations associated with negative stereotypes are well-known to induce subjective stress for the recipient (e.g., stereotype threat).

The present study assessed devaluation-related stress on health outcomes across several domains as well as global effects on overall well-being. Specifically, we examined the 
experiences of female students at a STEM institution where extensive statements of initiatives on inclusion and diversity exist. The findings suggest that despite extensive support for inclusion, implicit, negative biases towards women remain entrenched, resulting in overall negative reported experiences by female students. Related factors included greater perceived stress and low sense of control, greater feelings of objectification, and few positive exemplars. Interestingly, when examining factors that emerge for male students, factor loadings included less perceived stress, being treated as uneducated, and overall unfriendly treatment. Females experienced the burden of social devaluation, resulting in lower ratings of physical and mental health. These data suggest that such initiatives may not be addressing 1) implicit negative attitudes still pervasive for females, and 2) the internalization of such stigma for the recipient.

\section{Overt discrimination and health}

Robust associations exist between overt discrimination and physiological dysregulation including elevated blood pressure, higher resting heart rate, slower recovery to baseline levels after social stress, and cardiovascular illness (for reviews see Pascoe \& Richman, 2009; Jackson, Kubzansky \& Wright, 2006). Moreover, heightened physiological activity in the major stress systems has been associated with perceptions of discrimination and "weathering," that is, an additional burden on deleterious health outcomes (e.g., Geronimus, Hicken, Keene, \& Bound, 2006). The embedding of adversity due to more subtle and cumulative social evaluation has not been widely investigated, particularly in STEM populations where multiple inclusion initiatives exist.

Perceived stress and sex differences

A related literature has examined sex differences in perceptions of stress, although findings are inconsistent. Broadly, women report higher negative affect in response to 
psychosocial stressors (e.g., Kelly et al., 2008). For example, in a standard acute lab stress task with concomitant increases in salivary cortisol (i.e., the Trier Social Stress Test; Kirschbaum, Pirke \& Hellhammer, 1993; Kelly et al., 2008) no sex differences in cortisol were found. Subjectively, however, women experienced increased negative mood and negative personal evaluations of performance. In a related study on microaggressions in the workplace, participants read passages that depicted subtle sexist attitudes and blatant denials of harm by a supervisor. Women detected more discrimination, particularly when the sexist beliefs were more subtle (Basford, Offermann, \& Behrend, 2014).

In this study, it was hypothesized that the underlying constructs between discrimination, microaggressions, and personal stress would reveal cumulative effects, and these effects would be associated with poorer health across several domains, including overall health, mental and physical health, and life satisfaction. To examine global indicators of devaluation on health, composites of social devaluation (SD) and total well-being (TWB) were created. Specifically, we hypothesized that females would report greater SD and lower TWB compared to male and Caucasian counterparts. Moreover, we expected that women would perceive the types of the stressful treatment differently which as explored with a factor analysis.

The present study adds to the literature on both overt and subtle devaluation experiences on health, particularly for young female scientists in STEM fields. Uniquely, these findings add that the subtle implicit biases and beliefs can influence multiple domains of health. Despite extensive initiatives for diversity and inclusion, these findings suggest intractable beliefs are untouched by such initiatives. These data warrant interventions titrated to more subtle and pervasive beliefs regarding female scientists. Research that attempts to explicate the devaluation 
mechanisms in poor health can aid in diverting the attrition rates for females in STEM and add to the future of inclusion practices.

\section{Method}

\section{Participants}

Participants were recruited online via SONA recruitment database for students and the community. A final sample included 292 participants from a private STEM university in the northeast, ages $18-23,55 \%$ female, and $20.5 \%$ minority. Respondents were awarded course credit for their participation.

\section{Sociodemographic}

Participants were asked their age (in years), sex, and college status (freshman-senior). Participants were asked their primary heritage group: American Indian or Alaska Native (0\%), Asian (13.4\%), Black or African American (2.7\%), Latin American (2.4\%), Native Hawaiian or Pacific Islander (0\%), White (71.9\%), or did not answer $(9.6 \%)$.

\section{Health Measures}

Participants were asked to rate their health over four domains using a Likert scale from one to four, where one $=$ poor, two $=$ average, three $=$ good, and four $=$ excellent The specific domains included mental and physical health, overall well-being, and life satisfaction.

\section{Stress Measures}

Perceived Stress Scale (PSS; Cohen \& Williamson, 1988). This is a reliable and validated measure of perceived stress over the past week, using a Likert scale from one-five, where one $=$ never, two $=$ almost never, three $=$ sometimes, four $=$ fairly often, and five $=$ very often. For example, "how often have you been upset because of something that happened unexpectedly?" 
Four items were reverse scored, before averaging, such that a higher endorsement reflected higher perceived stress (Cronbach $\alpha=0.87$ ).

Daily Discrimination (DD; Williams, 1999). This is a reliable and validated measure of perceived DD, using a Likert scale from zero-four, where zero $=$ never, one $=$ almost never, two $=$ sometimes, three =fairly often, and four = very often, for example, "how many times daily are you treated with suspicion?" Four items were reversed scored, before averaging, such that a higher endorsement reflected higher perceived stress (Cronbach $\alpha=0.86$ ).

Modified Microaggressions Scale (MMS; Nadal, et al., 2011). This is a reliable and validated measure of the frequency of microaggression experiences. The Likert scale is rated from zerofive, where zero $=$ did not experience, one $=$ about once a year, two $=$ about once every few months, three $=$ about once a week, four $=$ about once a week, five $=$ at least 3 times per day (Cronbach $\alpha=0.78$ ). The modification included adding an open-ended question after items where respondents were asked the primary reason they were treated unfairly for categories other than race. These included: sex, sexual orientation, age, weight, foreign-born status, or other. For example, "Someone assumed that I would not be intelligent because of my race, sex, sexual orientation, age, weight, foreign-born status, or other”.

Social Devaluation Composite. Z-scoring DD, PSS, and MMS constructed the composite. The mean of the scales was computed to create a measure of overall Social Devaluation (SD).

Total Well-being. The composite was constructed by z-scoring the four single health ratings: overall, general, physical health, and life satisfaction. The mean of the scales was computed to create a measure of Total Well-being (TWB).

\section{Results}


Data were first examined for normality and missing data. All variables were normally distributed and there were zero missing data points $(N=292)$ in the analysis. T-tests were used to analyze differences by sex (2). Hierarchical linear regression and univariate analyses of variance were conducted to examine the main effects and interactions between sex with each domainspecific measure of stress and the SD composite. Age and college status were entered as covariates. Table 1 describes the zero-order correlations, means, and standard deviations of the primary variables.

Table 1.

\begin{tabular}{|c|c|c|c|c|c|c|c|c|c|c|c|c|c|c|}
\hline Variable & $\mathrm{M}$ & $\mathrm{SD}$ & 1 & 2 & 3 & 4 & 5 & 6 & 7 & 8 & 9 & 10 & 11 & 12 \\
\hline 1. Age & 19.74 & 1.35 & - & & & & & & & & & & & \\
\hline 2. Sex & 0.45 & 0.49 & 0.06 & - & & & & & & & & & & \\
\hline 3. College Status & 2.31 & 1.19 & $.912 * *$ & 0.03 & - & & & & & & & & & \\
\hline 4. Race/Ethnicity & 3.72 & 0.91 & 0.05 & $.131^{*}$ & 0.01 & & & & & & & & & \\
\hline 5. Minority Status & 0.80 & 0.40 & 0.06 & 0.06 & 0.02 & $.960 * *$ & & & & & & & & \\
\hline \multicolumn{15}{|l|}{ Subjective Measures } \\
\hline 6. Daily Discrimination & 1.84 & 0.56 & 0.07 & -0.02 & -0.10 & 0.01 & 0.014 & & & & & & & \\
\hline 7. Microaggressions & 0.62 & 0.38 & -0.08 & $-.137 *$ & 0.04 & -0.03 & 0.01 & $.275^{* *}$ & & & & & & \\
\hline 8.. Percevied Stress & 2.84 & 0.64 & -0.08 & $-.167 * *$ & -0.08 & -0.05 & -0.043 & $.387 * *$ & 0.076 & & & & & \\
\hline 9.. SR health & 2.76 & 0.89 & 0.07 & 0.08 & 0.07 & 0.07 & 0.085 & $-.296 * *$ & 0.055 & $-.580 * *$ & & & & \\
\hline 10. SR mental & 2.50 & 1.00 & 0.05 & 0.07 & 0.05 & 0.04 & 0.033 & $-.233 * *$ & 0.017 & $-.479 * *$ & $.557 * *$ & & & \\
\hline 11. SR physical & 2.72 & 0.93 & 0.05 & 0.00 & -0.01 & 0.01 & 0.037 & $-.187 * *$ & -0.08 & $-.279 * *$ & $.459^{* *}$ & $.310^{* *}$ & & \\
\hline 12. Life Satisfaction & 2.76 & 0.88 & -0.03 & -0.07 & -0.02 & 0.04 & 0.016 & $-.235^{* *}$ & 0.061 & $-.532 * *$ & $.671 * *$ & $.532 * *$ & $.391 * *$ & \\
\hline 13. Social Devaluation* & 0.00 & 0.54 & 0.01 & $-.227 * *$ & -0.02 & -0.05 & -0.021 & $.687 * *$ & $.688 * *$ & $.380 * *$ & $-.235 * *$ & $-.163 * *$ & $-.176 * *$ & $-.161 * *$ \\
\hline
\end{tabular}

\section{Sex Differences}

The t-tests examining sex differences obtained significant results for PSS $(t(1,288)=$ $2.89, p<.05)$, and MMS $(t(1,289)=2.35, p<.05)$, and SD $(=t(1,289)=3.96, p<.001)$. Females reported higher experiences in Microaggressions $(\mathrm{M}=.557, \mathrm{~F}=.661)$ and Perceived Stress $(\mathrm{M}=$ 2.720, $\mathrm{F}=2.937)$, but not Daily discrimination $(p>.05)$.

\section{Perceived Stress}

The next models used hierarchical regression modeling to examine interactions of sex with each unique stress measure to predict health outcomes. There was a significant interaction 
between PSS by sex on physical health, controlling for age and minority status $(B=-0.391, t(4$, 262), $p<.05$; Figure 1).

Figure 1.

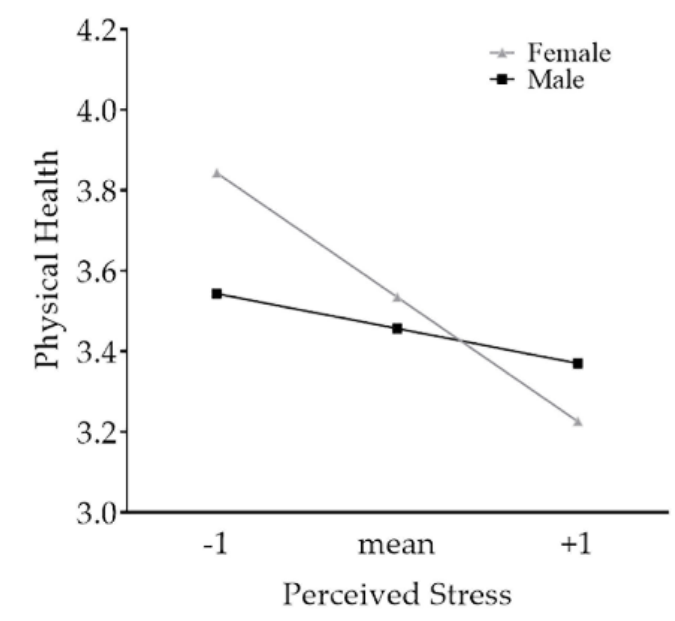

Females in the lower PSS rated their physical health higher than females in the higher stress groups, as well as males across all levels of stress. Each unit increase of PSS was associated with -0.391 decreases in physical health. When examining overall health, results obtained the main effect of PSS $(B=-0.623, t(4,262), p<.001)$, but the interaction term was n.s. Each unit increase of PSS was associated with -0.623 decreases in overall well-being. There was also a main effect for PSS on mental health, showing a similar trend $(B=-0.508, t(4,262)$, $p<.001)$. There were main effects for both sex $(B=-0.131, t(4,262), p<.05)$ and PSS $(B=-0.585$, $t(4,262), p<.001)$, on life satisfaction, but the interaction term was not significant. Females reported significantly lower life satisfaction and higher PSS.

\section{Daily Discrimination}

In the next models, sex by DD were examined and results obtained main effects for DD on all health measures: overall health: $B=-0.305, t(4,262), p<.001$, mental health $(B=-0.201, t(4$, 
262), $p<.001$, physical health: $B=-0.274, t(4,262), p<.001$, and life satisfaction: $B=-0.242, t(4$, 262), $p<.001$. Units higher in DD were associated with decreased health across all domains. There were no main effects by sex and the interaction was n.s.

\section{Principal Component Analysis}

A principal component analysis was conducted to assess the major factors that were influential on health ratings. These included 40 items from the perceived stress, microaggression, and discrimination scales. The initial extraction analysis yielded five factors that explained 50.22 of the variance. Inspection of the communalities and the scree plot revealed that three factors had the highest loadings and in the next iteration, the extraction was forced to yield three factors with a Varimax (oblimin) rotation and split the file to compare female to male factors. For females, factor 1 was loaded on more of the perceived stress items and low control (cannot handle personal problems, difficult to cope, no control, opinion was overlooked, no support, stressed); factor 2 was labeled as perceived social devaluation (people were surprised at my success, I was ignored, treated unfriendly, assumed inferior work); the final factor was labeled as positive exemplars found in TV, government, magazines, CEOs, books, and overall few positive portrayals). For males, a different pattern emerged: Factor 1 was similar in perceived stress, but for greater loadings on items related to treatment (treated with less respect, as not smart, with less courtesy); factor 2 was the positive exemplars; and factor 3 was loaded on different microaggressions items than females (assumed lower education, should not complain about issues; see Table 2). Independent t-tests were conducted to examine whether these factors were significantly different by sex. Results shows that factors 1 and 2 were significant $(t(1,288)=$ $7.055, p<.001 ; t(1,288)=4.483, p<.001)$.

Table 2 illustrates the results of the factor analysis and loadings by sex. 


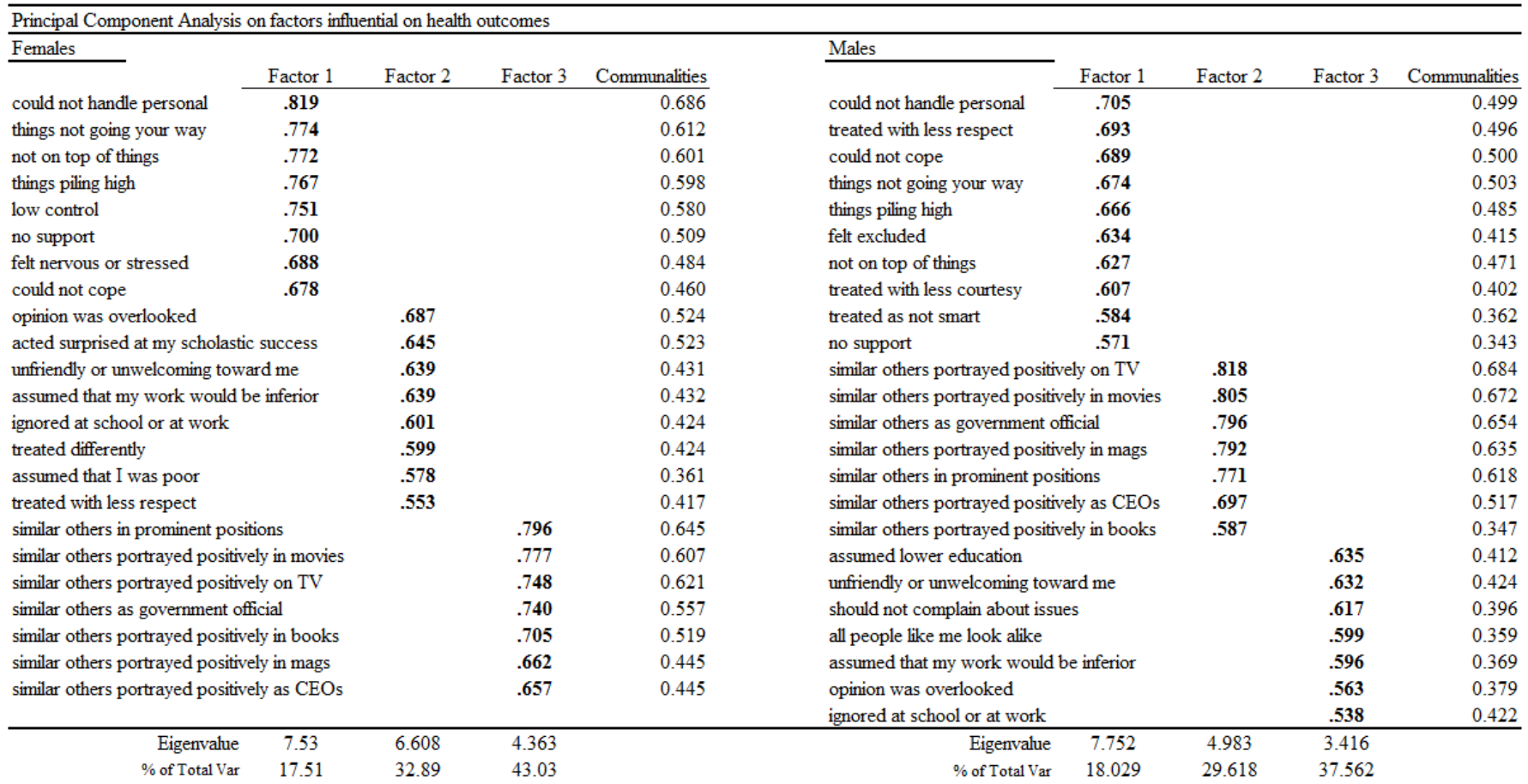

The EFA provides insights as to how males and females were perceiving mistreatment.

The constructs for females appear to be drive by internal evaluations and perceived stress (e.g., being overwhelmed, not feeling able to cope); whereas the first factors for males appeared to be about their treatment (e.g., treated with less respect).

\section{Discussion}

Taken together, these data suggest that for STEM-oriented females students, the extensive initiatives on diversity and inclusion are missing some critical element(s) that address entrenched beliefs about female stereotypes, internal evaluations, and the victim's inability to effectively address when such stressful events occur, which we call 'socialized acquiescence'.

The present study investigated the cumulative effects of devaluation and stress across several domains, including discrimination and microaggressions, on health and well-being. Interestingly, when examining unique domains of devaluation, robust effects emerged for females compared to their male counterparts. Females were higher across the domains of PSS, 
MMS, and DD, as well as the composite of overall SD. Relationships to health showed that females reported lowest overall health, mental and physical health, and life satisfaction. The interaction term of sex by PSS and sex by DD predicted health outcomes. Interestingly, MMS did not predict health in these data, despite being highly correlated with DD.

Given that these data show that female students still have stress and doubts about their inherent worth in STEM fields, and experience daily experiences of being devalued, further research can explore more specific interventions and tools for these specific concerns. EFA

Interestingly, the way females and males perceived microaggressions were loaded onto different factors. This could speak to the literature that females evaluate themselves more poorly and experience higher distress in the college experience (e.g., Pomerantz, Altermatt, \& Saxon, 2002) and stereotype threat, particularly for subtle negative cues (e.g., Nguyen \& Ryan, 2008). This suggests that part of any effective academic intervention would be a focus on these internal evaluations, as well as the different stressors for females and males in college.

Overall, enhancing female students' experiences of status, worth, equality, and support in STEM education will include implementing new methods that work on multiple fronts: 1) empower students to overcome challenges across several domains, including psychosocial factors that enhance psychological health (Smith, Arlotta \& Watt, 2015); 2) address negative implicit biases in the larger academic culture; and 3) assess students' actual personal evaluations. There is little available literature to investigate the psychological and physiological benefits of students organizations; however, STEM universities in particular could receive help from more initiatives where all members of the academic community participate, including males, staff, and 
faculty to address ingrained beliefs about female contributions and changes in university culture. It is not clear in these data where the experiences are occurring.

These data can inform current initiatives and programs designed to increase inclusion and diversity and decrease stereotypes; however, it may be that implicit stereotype threat could be a specific focus for more effective initiatives, in order to give students psychological resources and preparedness for when they perceive subtle SD. By making these processes explicit, we can not only substantially improve students' overall well-being, but increase actual inclusion and value of diverse populations. Moreover, the benefits of this work include assessing physiological measures as objective mechanisms in the widening health disparities for those who bear a disproportion of these burdens.

\section{Future directions}

In the next iteration, it would be useful to add physiological and anthropometric measures as early indicators of metabolic syndrome, vital exhaustion, or chronic illnesses to examine the relationships between SD and cumulative stress on the major stress and health systems.

Moreover, extending the age range of the sample, recruiting from community populations, and adding indices of diagnosed health issues can give us further insight on how these negative social experiences shape health over the lifespan. Given that health disparities are widening in the United States, it may be that chronic devaluation for women is an entrenched social mechanism that influences health over time.

\section{Limitations}

There are limitations in the present study that warrant discussion. Primarily, these data are self-reported, which can be subject to reporting biases depending on the availability heuristic, that is, if a person just experienced a microaggression or currently feels negative, recent 
experiences or perceptions may skew the reports. As stated above, next iterations can include objective measures of health, including diagnoses of acute and/or chronic illnesses, as well as biomarkers such as salivary and hair cortisol, and related measures of physiological chronic stress. Moreover, the diversity of the sample can be improved by oversampling for race/ethnic minorities, as well as foreign-born individuals. The present study examined specific domains of both social stressors and health. Additional measures can include other types of potential sources of stress that influence health. Finally, these data are drawn from a relatively young sample of 18-23-year-old college students. In order to assess these measures as predictors of health disparities, a wider range of age, community participation, and diversity could improve the predictive outcomes. 


\section{References}

Adler, N. E., \& Rehkopf, D. H. (2008). U.S. disparities in health: Descriptions, causes, and mechanisms. Annual Review of Public Health, 29, 235-252.

Balsam, K. F., Molina, Y., Beadnell, B., Simoni, J., \& Walters, K. (2011). Measuring multiple minority stress: the LGBT People of Color Microaggressions Scale. Cultural Diversity and Ethnic Minority Psychology, 17(2), 163.

Basford, T. E., Offermann, L. R., \& Behrend, T. S. (2014). Do you see what I see? Perceptions of gender microaggressions in the workplace. Psychology of Women Quarterly, 38(3), 311-326.

Cohen, S. \& Williamson, G. (1988). Perceived stress in a probability sample of the U.S. In S. Spacapam \& S. Oskamp (Eds.), The social psychology of health: Claremont Symposium on Applied Social Psychology. Newbury Park, CA: Sage.

Geronimus, A. T., Hicken, M., Keene, D., \& Bound, J. (2006). "Weathering" and age patterns of allostatic load scores among blacks and whites in the United States. American Journal of Public Health, 96(5), 826-833.

Jackson, B., Kubzansky, L., \& Wright, R. J. (2006). Linking perceived unfairness to health: The Perceived Unfairness Model. Review of General Psychology, 10(1), 21-40.

Johnson, K.A., Johnson, W.M., Thomas, J.M., \& Green, J.J. (2018). Microaggressions at the University of Mississippi. http://socanth.olemiss.edu/wpcontent/uploads/sites/154/2018/10/Microaggressions-report-10-9-18.pdf

Kelly, M. M., Tyrka, A. R., Anderson, G. M., Price, L. H., \& Carpenter, L. L. (2008). Sex differences in emotional and physiological responses to the Trier Social Stress Test. Journal of Behavior Therapy and Experimental Psychiatry, 39(1), 87-98. 
Kirschbaum, C., Pirke, K. M., \& Hellhammer, D. H. (1993). The 'Trier Social Stress Test'-a tool for investigating psychobiological stress responses in a laboratory setting. Neuropsychobiology, 28(1-2), 76-81.

Major, B., Mendes, W. B., \& Dovidio, J. F. (2013). Intergroup relations and health disparities: A social psychological perspective. Health Psychology, 32(5), 514.

Miner, K. N., Settles, I. H., Pratt-Hyatt, J. S., \& Brady, C. C. (2012). Experiencing Incivility in Organizations: The Buffering Effects of Emotional and Organizational Support. Journal of Applied Social Psychology, 42(2), 340-372.

Nadal, K. L. (2011). The Racial and Ethnic Microaggressions Scale (REMS): construction, reliability, and validity. Journal of Counseling Psychology, 58(4), 470.

Nguyen, H. H. D., \& Ryan, A. M. (2008). Does stereotype threat affect test performance of minorities and women? A meta-analysis of experimental evidence. Journal of Applied Psychology, 93(6), 1314.

O'Brien, K. M., Tronick, E. Z., \& Moore, C. L. (2013). Relationship between hair cortisol and perceived chronic stress in a diverse sample. Stress and Health, 29(4), 337-344.

O’Brien, K. M., Meyer, J., Tronick, E., \& Moore, C. L. (2017). Hair cortisol and lifetime discrimination: Moderation by subjective social status. Health Psychology Open, 4(1), 2055102917695176

Pascoe, E. A., \& Smart Richman, L. (2009). Perceived discrimination and health: A metaanalytic review. Psychological Bulletin, 135(4), 531-554.

Pomerantz, E. M., Altermatt, E. R., \& Saxon, J. L. (2002). Making the grade but feeling distressed: gender differences in academic performance and internal distress. Journal of Educational Psychology, 94(2), 396. 
Smith, K. A., Arlotta, P., Watt, F. M., The Initiative on Women in Science and Engineering Working Group, \& Solomon, S. L. (2015). Cell Stem Cell, 16(3), 221-224.

Smith, W. A., Hung, M., \& Franklin, J. D. (2011). Racial battle fatigue and the miseducation of Black men: Racial microaggressions, societal problems, and environmental stress. The Journal of Negro Education, 63-82.

Stern J., Reid, E., Bancroft, K. (2015). Teaching Introductory Computer Science for a Diverse Student Body: Girls Who Code Style. Proceedings of the 46th ACM Technical Symposium on Computer Science Education.

Sue, D. W., Capodilupo, C. M., Torino, G. C., Bucceri, J. M., Holder, A., Nadal, K. L., \& Esquilin, M. (2007). Racial microaggressions in everyday life: implications for clinical practice. American Psychologist, 62(4), 271.

The State University of New York. (n.d.) Diversity Programs. https://www.suny.edu/diversity/diversity-programs/

Title IX and STEM. (2019). Research and Trends for Women in STEM. https://research.swe.org/title-ix-and-stem/

Troxel, W. M., Matthews, K. A., Bromberger, J. T., \& Sutton-Tyrrell, K. (2003). Chronic stress burden, discrimination, and subclinical carotid artery disease in African American and Caucasian women. Health Psychology, 22(3), 300-309.

Williams, D. R. (1999). Race, Socioeconomic Status, and Health: The Added Effects of Racism and Discrimination. Annals of the New York Academy of Sciences, 896, 173-188.

Women and STEM. (2017). Title IX at 45. Retrieved 14 March 2019, from https://www.ncwge.org/TitleIX45/Women\%20and\%20STEM.pdf 
Wong, G., Derthick, A. O., David, E. J. R., Saw, A., \& Okazaki, S. (2014). The what, the why, and the how: A review of racial microaggressions research in psychology. Race and Social Problems, 6(2), 181-200. 


\section{Figure Captions.}

Figure 1. Figure 1 illustrates the interaction between PSS and sex on physical health.

Females with lower perceived stress rated their physical health higher, compared to those females in higher perceived stress group. 